\title{
Refining the workflow of combustion chamber with toroidal recirculation mixing zone
}

\author{
Mikhail Orlov ${ }^{1}$, Vladislav Anisimov ${ }^{11}$, Oleg Kolomzarov ${ }^{1}$ and Mikhail Anisimov ${ }^{1}$ \\ Samara National Research University, 443086, Moskovskoye Shosse 34, Samara, Russia
}

\begin{abstract}
The object of the study is a straight-flow annular combustion chamber (CC) with toroidal recirculation-mixing zone which has significant reserves of minimization in size. The workflow of the $\mathrm{CC}$ has a number of problems, which arise due to the lack of experimental and calculated data. The aim of the study is to refine the design of the $\mathrm{CC}$ with toroidal recirculation-mixing to obtain the necessary workflow parameters. The first step of maturation involved the organization of swirl structure at primary zone. On the second step there were conducted the refining of the flame shape in primary combustion zone. The third step was devoted to the formation of the necessary temperature field at the outlet of the CC. As a result, an acceptable design of the combustion chamber was obtained.
\end{abstract}

\section{Introduction}

The design of the serial power plant was used as a prototype to create a new engine. In a new project the scheme solution of inlet duct and centrifugal compressor was not changed, but radial turbine became axial. This entailed a change in the combustion chamber. After working out a number of possible schemes, it was decided to choose a straight-flow combustion chamber (CC) of annular type with a toroidal recirculation-mixing zone, which has substantial reserves of minimization in size. A feature of this combustion chamber is its diagonal arrangement relative to the axis of the engine. There were several problems with the CC workflow, which are related to the lack of experimental and calculated data [1-6].

The aim of the paper is the maturation of the design of the combustion chamber with toroidal recirculation mixing zone for optimization its workflow.

To achieve this goal, it is necessary to solve the following tasks:

1) refine the aerodynamic flow structure to form a toroidal vortex in the primary combustion zone;

2) to form the combustion process in such a way that there is no local overheating of the flame tube wall;

3) provide the required temperature field at the outlet from the combustion chamber.

\footnotetext{
${ }^{1}$ Corresponding author: vradik@mail.ru
} 


\section{Methods}

The maturation of CC was carried out by simulation method in software package ANSYS Fluent. The scheme of the initial design of the combustion chamber is shown in Figure 1. Data of the mode parameters which are necessary to perform the simulation were taken from the results of the thermogasodynamic calculation.

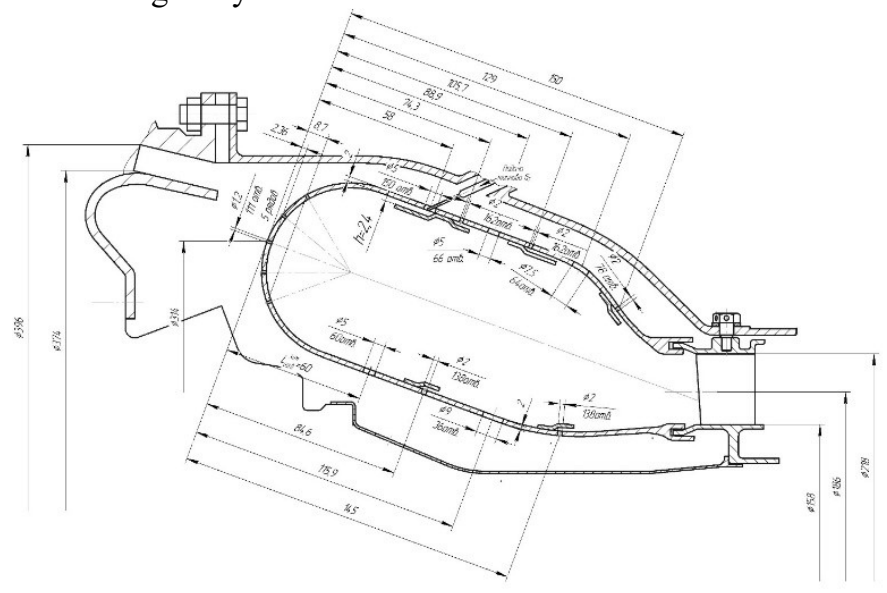

Fig. 1. The scheme of basic version of CC.

In the beginning, the sector of volume geometrical model of the basic version of $\mathrm{CC}$ was created (fig. 2, a). This sector corresponds to $120^{\circ}$ and includes four injectors.

On the basis of the geometric model, a grid of finite elements was generated (fig. 2, b). The number of elements of the grid model is 12.5 million. The skewness is 0.87 , which indicates the acceptable quality of the grid. A periodic condition was set on the side faces of the sector.

The choice of simulation models was determined by the accuracy of modeling the workflow in the combustion chamber [7,8]. The following calculation models were chosen: turbulent model - Reynolds Stress, radiation model - Discrete Ordinates, combustion model - Non-premixed, kinetic mechanism - Jeta 39. Kerosene was chosen as a fuel. The calculation was carried out in a steady state for the nominal operating mode of the engine. The temperature and total pressure of the air flow at the inlet were set as the boundary conditions, as well as the total pressure and gas flow rate at the outlet.

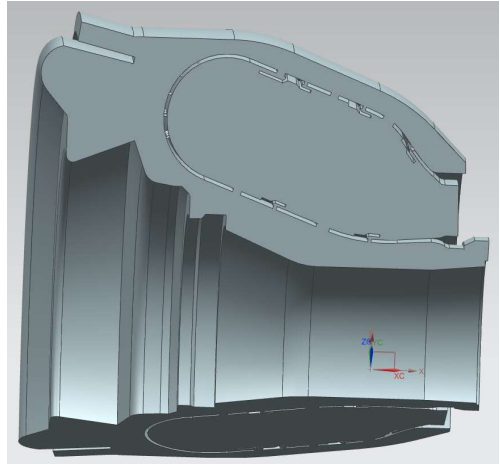

a

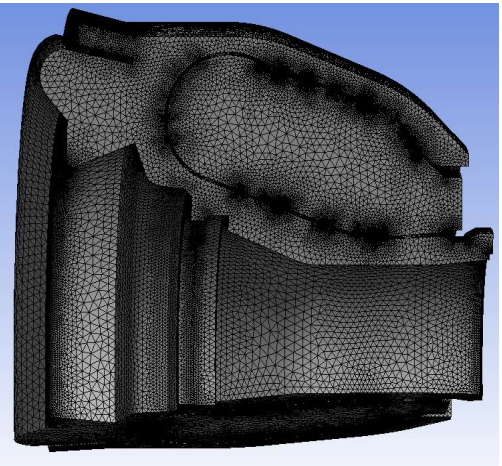

b

Fig. 2. The volume geometrical (a) and mesh (b) models of CC. 


\section{Refining}

\subsection{The first stage}

At the first stage, the refining of the flame tube design was carried out to organize the vortex structure in the primary zone.

On the vector field (fig. 3, a) obtained from simulation in the cross section of the nozzle, it can be seen that the design of the initial version does not allow us to give the required swirled flow. This results in insufficient mixing of the flow and afterburning of the mixture in the dilution and mixing zones. To obtain a stable and developed recirculation zone, it was suggested to use a protuberance on the inner part of the flame tube that performs the function of a "springboard". Due to the insufficient swirling of the flow in the primary zone, additional changes were made:

- the air supply holes were offset by $10 \mathrm{~mm}$ downstream, to increase the mixing path and evaporation of the fuel-air mixture.

- the angle of the holes on the front wall was changed. It was changed from the normal direction of the wall to 45 degrees to the normal, to create an air curtain and reduce the resistance to the flow that runs along the wall.

- the nozzle was moved to the air intake hole of the combustion zone into the gap between the flame tube and the deflector to increase the time for mixing the fuel and air and its evaporation.

- a protrusion was made on the inner part of the flame tube to direct the flow and its subsequent swirl.

- two rows of cooling holes were retained on the flame tube wall near the primary zone, but their diameters were reduced and the number was increased to preserve the total area of the holes on the wall of the toroidal part.

The result of the simulation obtained for the design after the introduction of the measures described above is shown in Figure 3. It clearly shows the formed structure of the stream recirculation in the primary combustion zone.

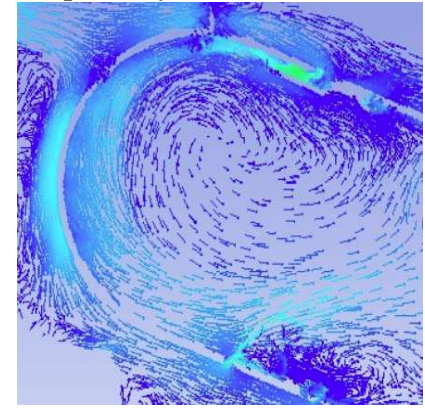

a

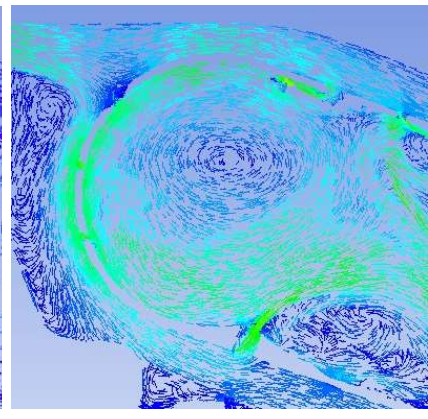

b

Fig. 3. Velocity vector field of the basic version (a) and after the first step (b).

\subsection{The second stage}

On the second step, the refining of the flame form in the primary zone was carried out. This zone should have sufficient swirled flow and should stabilize the flame within the recirculation-mixing zone. It will allow the fuel to burn almost completely in the primary zone and only a small amount of it will burn downstream. The original injectors were replaced with the jet nozzles with a spray angle of $12 . .15^{\circ}$ to form a stable and effective combustion zone. Blade vortexers were added in the gap between the deflector of the air 
intake holes and the flame tube in order to improve the mixing of fuel and air. Figure 4 shows the comparison of the temperature fields in the primary zone of basic version (fig. 4, a), after the first step of maturation (fig. 4, b) and after the second step (fig. 4, c).

When comparing the first two fields, it is seen that the insufficient formation of a swirl in the primary zone leads to the burning of the fuel in the downstream zones and, as a consequence, the combustible mixture is brought to the outer wall of the flame tube. This can cause its overheating and burnout.

After the second step of maturation the mixing of the mixture was improved and the flame was concentrate in the recirculation-mixing zone. The small flame areas appeared outside the primary zone due to stabilization behind rows of large holes, but the slope of the cooling holes allowed reducing the thermal load on the wall without increasing the resistance to the main flow (fig. 4, c).

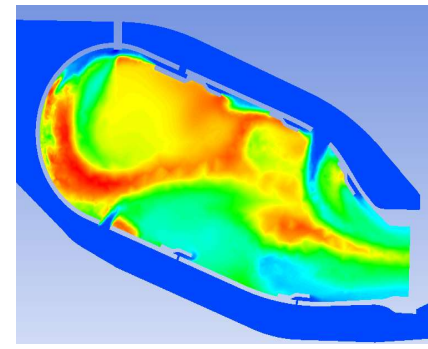

a

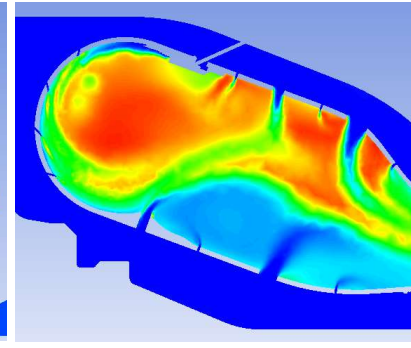

b

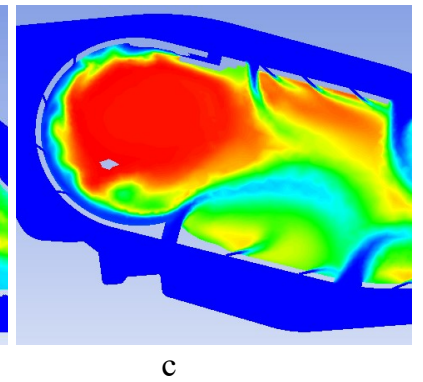

C

Fig. 4. The temperature fields at the cross section of injector of basic version (a), after the first step (b) and after the second step (c) of maturation.

\subsection{The third stage}

The third stage includes the formation of necessary temperature field at the outlet of CC. To reach the goal, the following events were carried out: make the necessary depth of penetration of air jets, change the number and location of the mixing holes.

As a result of these design changes, it was possible to obtain an acceptable temperature field distribution at the outlet of the CC (version 3.4, fig. 5, a). Since the change in the parameters forming the mixing zone is inherently associated with the air distribution in other areas of the $\mathrm{CC}$, when the area of the holes in the mixing zone was changed, the areas of the holes in the other zones were corrected. Figure 5 (b) shows the temperature field at the outlet of CC. As can be seen, the final version 3.4 does not have overheating zones from flame tongues at the outlet, which partially exist at other variants.

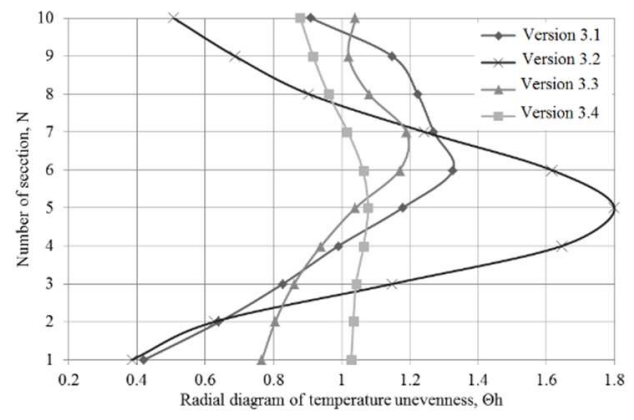

a

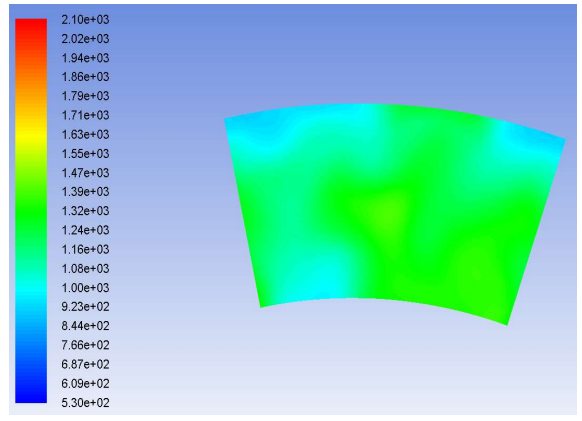

b

Fig. 5. Radial diagram of temperature unevenness (a) and the temperature field (b) at the outlet of CC. 


\section{Discussions}

Thus, it can be stated that as a result of the refining, an acceptable design of the combustion chamber was obtained.

The simulation methods allowed us to make the refining of CC design, organize the vortex structure and necessary flame form in the primary zone and temperature field at the outlet. Also, the use of computer simulation for the design refining has accelerated the process of getting a new $\mathrm{CC}$ design by about five times in comparison with the existing experience of traditional methods.

Based on the results of the work, the pilot sample of the CC was created by the methods of prototyping (laser sintering). The production cycle is reduced by at least 10 times compared with the use of traditional technologies.
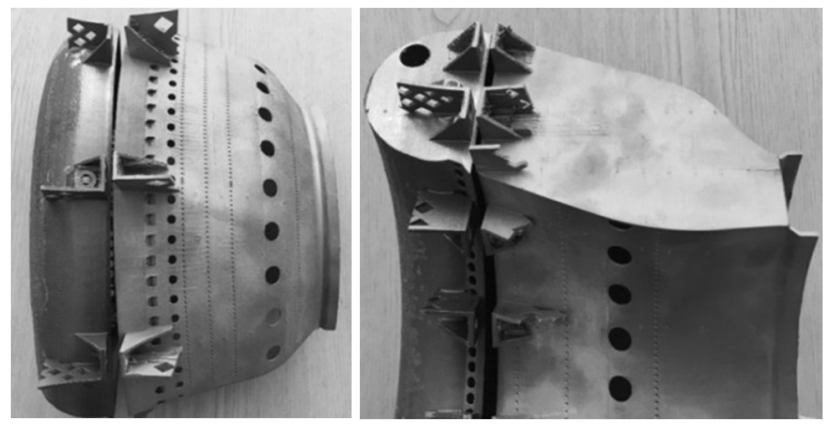

Fig. 6. The flame tube of developed combustion chamber.

Continuation of work occurs in the fire tests of the developed combustion chamber.

This work was supported by the Ministry of education and science of the Russian Federation in the framework of the implementation of the Program "Research and development on priority directions of scientific-technological complex of Russia for 20142020" (RFMEFI58716X0033).

\section{References}

1. A.M. Lansky, S.V. Lukachev, O.V. Kolomzarov. Vestnik Moskovskogo Aviatsionnogo Instituta, 23, 3, 47-57 (2016)

2. V.Y. Abrashkin Izvestiya Samarskogo nauchnogo tsentra Rossiyskoy akademii nauk, 8, 4, 1136-1141 (2006)

3. V.P. Lukachev, A.M. Lansky, V.Y. Abrashkin, A.A. Didenko, P.G. Zubkov, Y.L. Kovilov, S.G. Matveev, A.M. Tsiganov, M.A. Shamban, V.A. Yakovlev, Vestnik SGAU, 1, 11-39 (1999)

4. E. Carr, Futher applications of the lucas fan spray fuel injection combustion system // ASME, 1985. - P. 1-8.

5. E. Carr, H. Todd, The design and performance of a reverse flow combustion system for the TP 500 gas turbine engine, ASME, 1-6 (1989).

6. Carr E. The combustion of a range of distillate fuels in small gas turbine engines, ASME, 1-9 (1979).

7. M.Y. Orlov, V.M. Anisimov. Vestnik Moskovskogo Aviatsionnogo Instituta, 24, 2, 50-56 (2017)

8. V.M. Anisimov, M.Y. Orlov, I.A. Zubrilin. Vestnik Moskovskogo Aviatsionnogo Instituta, 23, 3, 29-39 (2016) 